\author{
Industrual BIO-TEST Laboratoried, Inc. \\ IEIO FRONTAGE ROAD \\ NORTHBROOK, ILLINOIS 60062
}

l.

STATUS REPORT TO

\title{
ATOMIC ENERGY COMMISSION
}

THREE-GENERATION REPRODUCTION STUDY WITH LOW DOSE IRRADIATED PQPDAAS

IN ALBINO RATS

Second Generation

CONTRACT NO. AT(11-1)-2014

MAY 10, 1971

IBT NO, B7041

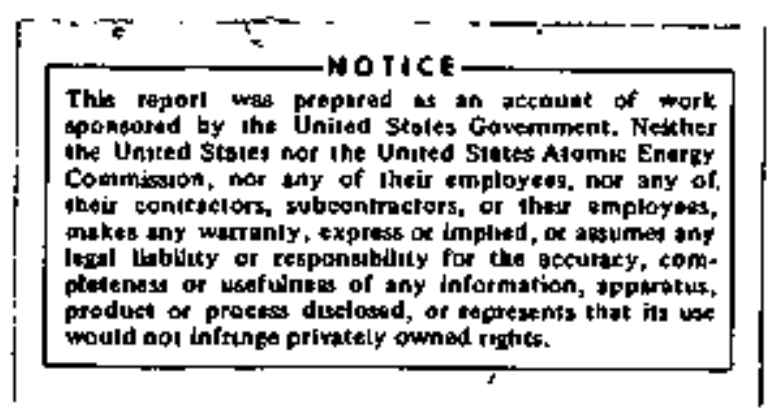




\section{DISCLAIMER}

This report was prepared as an account of work sponsored by an agency of the United States Government. Neither the United States Government nor any agency Thereot, nor any of their employees, makes any warranty, express or implied, or assumes any legal liability or responsibility for the accuracy, completeness, or usefulness of any information, apparatus, product, or process disclosed, or represents that its use would not infringe privately owned rights. Reference herein to any specific commercial product, process, or service by trade name, trademark, manufacturer, or otherwise does not necessarily constitute or imply its endorsement, recommendation, or favoring by the United States Government or any agency thereof. The views and opinions of authors expressed herein do not necessarlly state or reflect those of the United States Government or any agency thereof. 


\section{DISCLAIMER}

Portions of this document may be illegible in electronic image products. Images are produced from the best available original document. 


\section{Industrial B I0-TEST Laboratoried, Inc. 1810 FRONTAGE ROAD \\ NORTHBROOK. ILLINOIS 60062}

May 10,1971

Mr. Harold N, Miller, Director

Contracts Division

United States Atomic Energy Commission

9800 South Cass Avenue

Argonne, Ilinais 60439

Dear Mr. Miller:

Re: IBT No. B7041 - Three Generation Reproduction Study with Low Dose Irradiated Papayas in Albino Rats - Contract No. AT(11-1)-2014

We are submitting herewith our laboratory status report dated May 10, 1971, prepared in connection with the above study.

This report presents the results of the second generation.

Very truly yours,

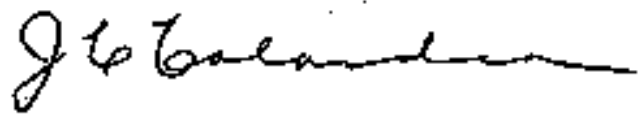

J. C. Calandra

President

$\mathrm{JCC} / \mathrm{kjl}$ 


\author{
STATUS REPORT TO \\ ATOMIC ENERGY COMMISSION \\ THREE-GENERATION REPRODUCTION STUDY WITH \\ LOW DOSE IRRADLATED PAFAYAS \\ IN ALBINO RATS \\ Second Generation \\ CONTRACT NO. AT(1)-1)-2014 \\ MAY 10, 1971 \\ IBT NO. B7041
}

I. Outline of Investigation:

A. Type and Length:

B. Animal Species Tested:

C. Starting Date:

D. Status of this Report:

E. Organization:
Three-Generation Reproduction Study

Charles River Strain Albino Rats

May 28, 1969

Completion of the Second Generation (Fl Parents - F $2 a$ and F2b Progeny)

A structural outline of the experiment is given in Table $I$. 


\section{TABLE I}

TEST MATERIAL: Irradiated Papayas

Three-Generation Reproduction Study - Albino Rats

Outline of Experiment

F1 Generation

\begin{tabular}{|c|c|c|c|}
\hline \multirow[b]{2}{*}{ Group } & \multirow[b]{2}{*}{ Treatment } & \multicolumn{2}{|c|}{ Number of Animals } \\
\hline & & Male & Female \\
\hline $\mathrm{c}$ & None & 50 & 50 \\
\hline $\mathrm{PC}$ & Non-irradiated papayas & 50 & 50 \\
\hline T-I & Irradiated papayas (75 krads) & 50 & 50 \\
\hline $\mathbf{T}=\mathrm{Il}$ & Irradiated papayas $(200 \mathrm{krads})$ & 50 & 50 \\
\hline
\end{tabular}

Papayas were 15 percent (wet weight) of the total diet.

F. Means of Administration: Voluntary Oral Ingestion 


\section{Summary}

A combination three-generation reproduction and two-year chronic oral toxicity study with albino rats being fed diets containing low dose irradiated papayas is being conducted. The results of the second generation of the reproductive portion revealed the following:

\section{A. Progeny (F2a and F2b)}

The number of pups delivered viable and the number retained at various points throughout lactation were normal for all groups.

Survival indices were essentially the same for treated and control progeny. The lactation index for the second litter of both test groups I and II was slightly lower than the control. However, these indices were within the normal range for the albino rat.

The body weight data obtained at weaning disclosed no significant differences between test and control values. 


\section{Parental Animals}

No effects which could be attributed to the ingestion of low dose irradiated papayas were noted in body weight gain, survival, mating and ferility indices, gestalion times or incidences of pregnancy and parturition.

Respectfully submitted,

INDUSTRLAL BIO-TEST LABORATORIES, INC.

Report prepared by:

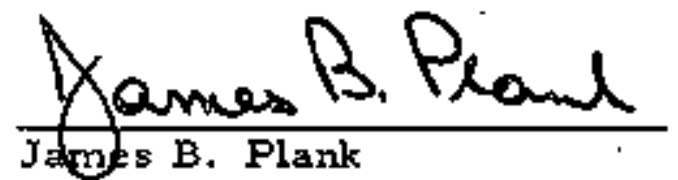

Senior Group Leader

Rat Toxicity

Report approved by:

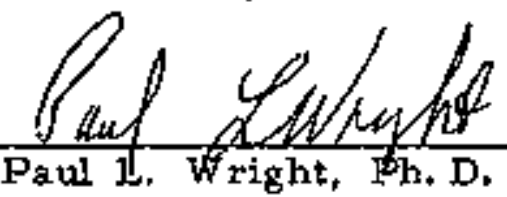

Section Head, Toxicology

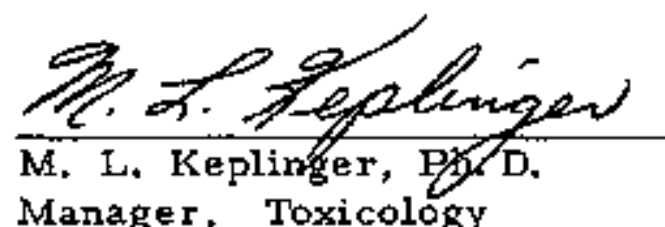

Manager, Toxicology

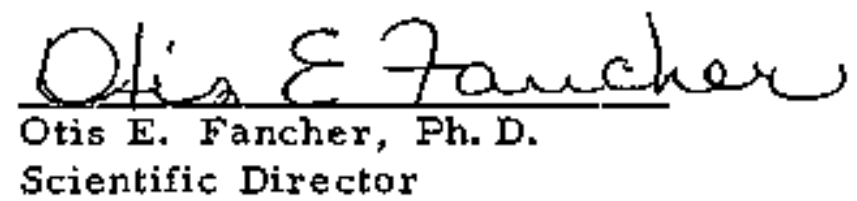

May $10,197 \mathrm{I}$

hp;kjl:psh 
III. Results

A. Parental Arimals

1. Boty Weights

Weekly body weight data for the first 72 days of the feeding period are presented in Tables II and III.

Mean body weights of females at Lays 1 and 21 of lactation are presented in Table IV. 
TABLE II

TEST MATERIAL: Irradiated Papayas

Three-Gene ration Reproduction Study - Albino Rats

Mean Body Weight and Weight Gain Data

Parental Male Rats

\begin{tabular}{|c|c|c|c|c|c|c|c|c|c|c|c|c|}
\hline Group & 0 & 1 & 2 & 3 & $\begin{array}{c}\mathrm{Bc} \\
\mathrm{We} \\
4\end{array}$ & $\begin{array}{c}\text { Weigh } \\
\text { Nurnbe } \\
5\end{array}$ & $\begin{array}{c}\text { rams) } \\
6\end{array}$ & 7 & 8 & 9 & 10 & $\begin{array}{c}\text { Total } \\
\text { Weight } \\
\text { Gain (grams) }\end{array}$ \\
\hline Control & 114 & 161 & 214 & 264 & 302 & 327 & 356 & 386 & 412 & 430 & 449 & 335 \\
\hline $\begin{array}{l}\text { Positive } \\
\text { Control }\end{array}$ & 109 & 153 & 200 & 249 & 284 & 318 & 347 & 374 & 398 & 418 & 435 & .326 \\
\hline$T-I$ & 116 & 160 & .207 & 255 & 295 & 312 & 345 & 371 & 402 & 417 & 436 & 320 \\
\hline I -II & 110 & 159 & 206 & 254 & 296 & 315 & 344 & 372 & 402 & 416 & 432 & 322 \\
\hline
\end{tabular}


TABLE Шr

TEST MATERIAL: Irradiated Papayas

Three-Generation Reproduction Study - Albino Rats

Mean Body Weight and Weight Gain Data

Parental Female Rats

\begin{tabular}{|c|c|c|c|c|c|c|c|c|c|c|c|c|}
\hline & & & & & Mean $\mathrm{I}$ & Weis & gran & & & & & $\begin{array}{c}\text { Total } \\
\text { Weight }\end{array}$ \\
\hline Group & 0 & 1 & 2 & 3 & 4 & 5 & 6 & 7 & 8 & 9 & 10 & Gain (grams) \\
\hline Control & 102 & 134 & 160 & 182 & 196 & 208 & 226 & 236 & 244 & 252 & 258 & 156 \\
\hline $\begin{array}{l}\text { Positive } \\
\text { Control }\end{array}$ & 100 & 134 & 160 & 184 & 198 & 211 & 222 & 231 & 245 & 254 & 262 & 162 \\
\hline$T-I$ & 108 & 135 & 160 & 182 & 196 & 216 & 224 & 234 & 248 & 253 & 261 & 153 \\
\hline $\mathrm{T}-\mathrm{II}$ & 101 & 134 & 166 & 188 & 204 & 221 & 228 & 236 & 244 & 253 & 248 & 147 \\
\hline
\end{tabular}


TABLE IV

TEST MATERIAL: Irradiated Papayas

Three-Generation Reproduction Study - Albino Rats

Mean Female Lactation Body Weights

\begin{tabular}{|c|c|c|c|c|}
\hline Group & Litter & \multicolumn{3}{|c|}{ Mean Female Body Weights (grams) } \\
\hline \multirow[t]{2}{*}{$\mathrm{C}$} & $\mathbf{F} 2 \mathbf{a}$ & 303 & & 310 \\
\hline & $\mathbf{F} 2 \mathbf{b}$ & 355 & & 333 \\
\hline \multirow[t]{2}{*}{ PC } & $F 2 a$ & 301 & $\cdot$ & 305 \\
\hline & $\mathbf{F} 2 \mathbf{b}$ & 353 & & 317 \\
\hline \multirow[t]{2}{*}{$T-1$} & F Za & 301 & & 300 \\
\hline & $\mathbf{F} 2 \mathbf{b}$ & 350 & & 316 \\
\hline \multirow[t]{2}{*}{$\mathrm{T}=\mathrm{II}$} & $\mathbf{F} 2 \mathrm{a}$ & 304 & & 314 \\
\hline & F $2 b$ & 352 & & 329 \\
\hline
\end{tabular}


2. Mortality

The mortality data are presented in Tables $V$ and VI.

\section{Reactions}

No untoward reactions were noted among the parental animals during the investigation. 
TABLE V

TEST MATERLAL: Irradiated Papayas

Three-Generation Reproduction Study - Albino Rats

Mortality Dața

Parental Generation Males

\begin{tabular}{|c|c|c|c|c|c|c|c|}
\hline \multicolumn{4}{|c|}{ First Mating } & \multicolumn{3}{|c|}{ Se cond Mating } & \\
\hline Group & Pre-M & $\mathrm{M}$ & Post-M & $\mathrm{M}$ & Post-M & Total & \\
\hline $\mathscr{H}$ & & & & & & & \\
\hline C & 0 & 0 & 0 & 0 & 0 & 0 & . \\
\hline$P C$ & 0 & 0 & 0 & 0 & 0 & 0 & \\
\hline$T-I$ & 0 & 0 & 0 & 0 & 0 & 0 & $\cdot$ \\
\hline T-II & 0 & 0 & 0 & 0 & 0 & 0 & \\
\hline
\end{tabular}

Pre-M = Pre-Mating Period

$M=$ Mating Period

Post-M = Post-Mating Period 
TABLE VI

TEST MATERLAL: Irradiated Papayas

Three-Generation Reproduction Study - Albino Rats

Mortality Data

Parental Generation Females

\begin{tabular}{|c|c|c|c|c|c|c|c|c|c|c|c|c|}
\hline \multirow[b]{2}{*}{ Group } & \multicolumn{6}{|c|}{ First Mating } & \multicolumn{6}{|c|}{ Second Mating } \\
\hline & Pre-M & $\mathrm{M}$ & $\mathrm{G}$ & $\mathrm{P}$ & $\mathrm{L}$ & $\mathbf{R}$ & $\mathrm{M}$ & $\mathrm{G}$ & $\mathbf{P}$ & $\mathbf{L}$ & Post-W & Tota1 \\
\hline C & 0 & 1 & 0 & 0 & 0 & 0 & 1 & 0 & 1 & 1 & 1 & 5 \\
\hline$P C$ & 0 & 0 & 0 & 0 & 0 & 0 & 1 & 0 & 0 & 1 & 1 & 3 \\
\hline$T-I$ & 0 & 0 & 0 & 0 & 0 & 0 & 0 & 0 & 2 & 0 & 1 & 3 \\
\hline T-II & 0 & 0 & 0 & 0 & 0 & 0 & 0 & 0 & 0 & 0 & 0 & 0 \\
\hline
\end{tabular}

Pre-M = Pre-Mating Period

$M=$ Mating Period

$G=$ Gestation Period

$P=$ Parturition

$L=$ Lactation Period

R = Resting Period

Post-W $=$ Post - Weaning Period 
4. General Data

General data for parental animals are presented in

Table VIf.

The indices employed ate defined below:

Mating Index $=\frac{\text { Number of Copulations }}{\text { Number of Estrus Cycles Required }} \times 100$

Fertility Index $=\frac{\text { Number of Females Becoming Pregnant }}{\text { Number of Copulations }} \times 100$

Incidence of Pregnancy $=\frac{\text { Number of Pregnancies }}{\text { Number of Females Mated }} \times 100$

Incidence of Parturition $=\frac{\text { Number of Parturitions }}{\text { Number of Pregnancies }} \times 100$ 
TABEE VII

TEST MATERIAL: Irradiated Papayas

Three-Generation Reproduction Study - Albino Rats

General Data - Parental Animals

Reproduction Performance

\begin{tabular}{|c|c|c|c|c|c|c|c|c|c|c|c|}
\hline \multirow[b]{2}{*}{ Group } & \multirow[b]{2}{*}{ Litter } & \multirow{2}{*}{$\begin{array}{r}\text { Mating } \\
\text { Fraction } \\
\end{array}$} & \multirow{2}{*}{$\begin{array}{l}\text { Index } \\
\text { Percent }\end{array}$} & \multicolumn{2}{|c|}{$\begin{array}{c}\text { Incidence of } \\
\text { Pregnancy }\end{array}$} & \multicolumn{2}{|c|}{$\begin{array}{l}\text { Fertility } \\
\text { Index }\end{array}$} & \multicolumn{2}{|c|}{$\begin{array}{c}\text { Incidence of } \\
\text { Parturition }\end{array}$} & \multicolumn{2}{|c|}{$\begin{array}{c}\text { Mean Gestation } \\
\text { Time (Days) }\end{array}$} \\
\hline & & & & Fraction & Percent & Fraction & Percent & Fraction & Percent & Mean & Range \\
\hline \multirow{2}{*}{ Control } & $\mathbf{F} 2 \mathrm{a}$ & $54 / 72$ & 75.0 & $49 / 50$ & 98.0 & $49 / 54$ & 90.7 & $48 / 49$ & 97.9 & 21 & $19-23$ \\
\hline & F2b & $50 / 68$ & 73.5 & $47 / 50$ & 94.0 & $47 / 50$ & 94.0 & $46 / 47$ & 97.9 & 22 & 20.23 \\
\hline \multirow[t]{3}{*}{$\begin{array}{l}\text { Positive } \\
\text { Control }\end{array}$} & $\mathrm{FZa}$ & $61 / 66$ & 92.4 & $49 / 50$ & 98.0 & $49 / 61$ & 80.3 & $48 / 49$ & 97.9 & 22 & $19-23$ \\
\hline & F2b & $53 / 62$ & 85.5 & $46 / 49$ & 93.9 & $46 / 53$ & 86.8 & $46 / 46$ & 100.0 & 22 & $19-23$ \\
\hline & F2a & $50 / 55$ & 90.9 & $49 / 49$ & 100.0 & $49 / 50$ & 98.0 & $49 / 49$ & 100.0 & 21 & $19-23$ \\
\hline \multirow[t]{3}{*}{$T=I$} & & & ing & & & & & & & & \\
\hline & F2b & $52 / 62$ & 83.9 & $49 / 50$ & 98.0 & $49 / 52$ & 94.2 & $49 / 49$ & 100.0 & 22 & $20-23$ \\
\hline & $F^{2} \mathrm{a}$ & $58 / 85$ & 68.2 & $48 / 50$ & 96.0 & $48 / 58$ & 82.8 & $48 / 48$ & 100.0 & 22 & $19-23$ \\
\hline $\mathbf{T}-\mathbf{I I}$ & F2b & $53 / 64$ & 82.8 & $49 / 50$ & 98.0 & $49 / 53$ & 92.4 & $49 / 49$ & 100.0 & 21 & $19 \cdot 23$ \\
\hline
\end{tabular}


B. Progeny

1. Population Data

Population data for the F 2 a and F 2 b litters are presented in Table VIII.

All pups delivered in both litters appeared outwardly normal and displayed normal growth through their lactation period. At weaning, an examination of each pup for external abnormalities was conducted and all pups were judged to be free of gross external abnormalities. The pups from females that died during the lactation period were sacrificed and are not included in the means or in calculating the lactation indices. 
TAELE VIII

TEST MATERIAL: Irradiated Papayas

Three-Generation Reproduction Study - Albino Rats

Population Data

\section{Progeny}

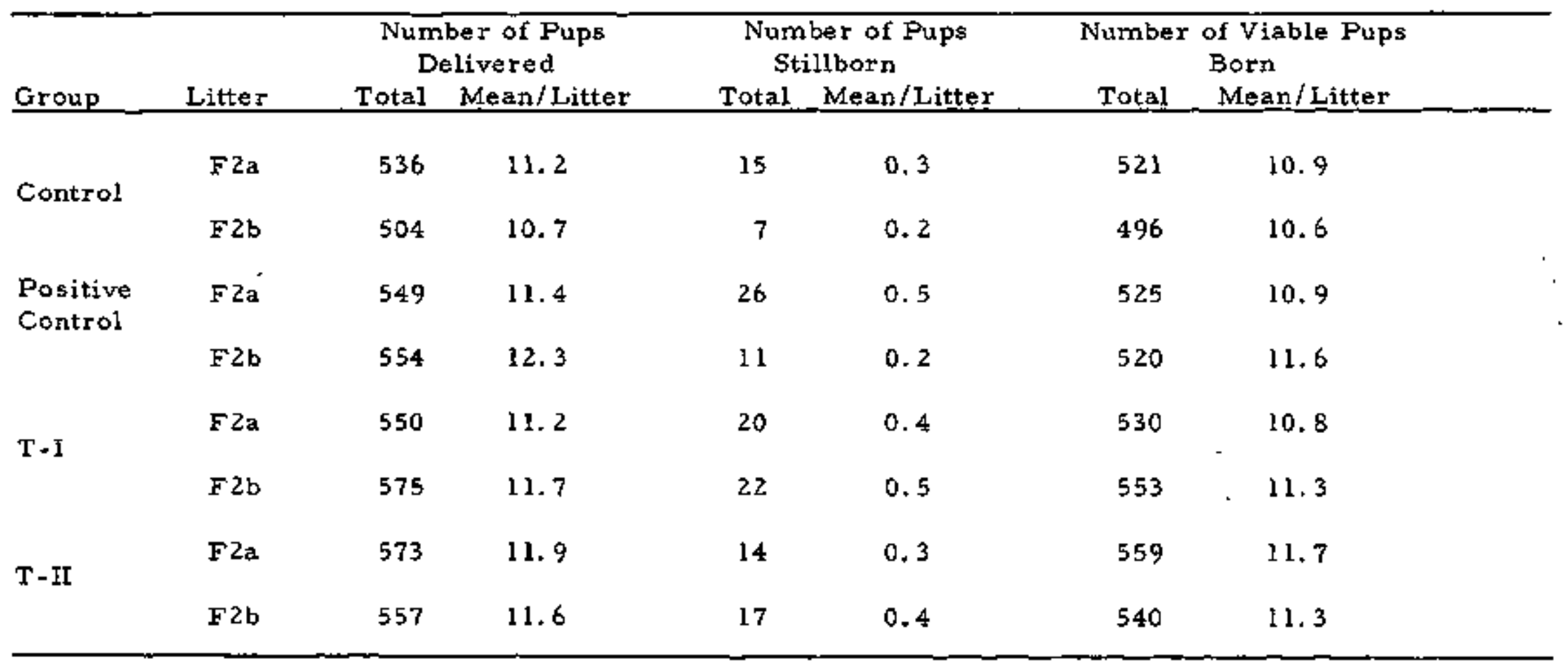


IABLE VIII continued

TEST MATERIAL: Irradiated Papayas

Three-Generation Reproduction Study - Albino Rats

Population Data

Progeny

\begin{tabular}{|c|c|c|c|c|c|c|c|c|c|c|c|}
\hline Group & Litter & \multicolumn{2}{|c|}{$\begin{array}{l}\text { Number of Pups } \\
\text { at Lactation Day } 1\end{array}$} & \multicolumn{5}{|c|}{$\begin{array}{l}\text { Number of Pups Number of Pups Retained } \\
\text { at Lactation Day } 4 \quad \text { at Lactation Day } 4\end{array}$} & \multicolumn{3}{|c|}{ Number of Weanlings } \\
\hline \multirow{2}{*}{ Control } & $\mathrm{FZa}$ & 500 & 10.4 & 473 & 9.9 & 341 & 7.1 & 153 & 153 & 306 & 6.4 \\
\hline & F2b & 488 & 10.4 & 469 & 9.9 & 345 & 7.3 & 170 & 142 & 312 & 6.6 \\
\hline \multirow{2}{*}{$\begin{array}{l}\text { Positive, } \\
\text { Control }\end{array}$} & F2a & 481 & 10.0 & 467 & 9.7 & 326 & 6.8 & 146 & 144 & 290 & 6.0 \\
\hline & $\mathrm{F} 2 \mathrm{~b}$ & 520 & 11.6 & 493 & 11.0 & 343 & 7.6 & 145 & 135 & 280 & 6.2 \\
\hline \multirow{2}{*}{$T-I$} & $F 2 a$ & 496 & 10.1 & 462 & 9.4 & 339 & 6.9 & 154 & 163 & 317 & 6.5 \\
\hline & $F 2 b$ & 542 & 11.1 & 533 & 10.9 & 364 & 7.4 & 141 & 126 & 267 & 5.5 \\
\hline \multirow{2}{*}{$T-I I$} & $\mathrm{F2a}$ & 535 & 11.2 & 492 & 10.3 & 346 & 7.2 & 146 & 171 & 317 & 6.6 \\
\hline & $\mathrm{F} 2 \mathrm{~b}$ & 515 & 10.7 & 497 & 10.4 & 351 & 7.3 & 134 & $\$ 45$ & 279 & 5.8 \\
\hline
\end{tabular}


2. Surviva1 Dala

Survival data for progeny are presented in Table IX. The number of pups per female was reduced to eight, four males and four females whenever possible, on the fourth day of the lactation period. 
TABLE IX

TEST MATERIAL: irradiated Papayas Three-Generation Reproduction Study - Albino Rats

Survival Indices

\begin{tabular}{lccccc}
\multicolumn{5}{c}{ Progeny } & \\
\hline Group & Litter & $\begin{array}{c}\text { Live Birth } \\
\text { Index }\end{array}$ & $\begin{array}{c}\text { 24-Hour } \\
\text { Surival } \\
\text { Index }\end{array}$ & $\begin{array}{c}\text { 4-Day } \\
\text { Survival } \\
\text { Index }\end{array}$ & $\begin{array}{c}\text { Lactation } \\
\text { Index }\end{array}$ \\
\hline Control & F2a & 97.2 & 96.0 & 90.8 & 89.7 \\
Positive & F2a & 95.6 & 98.4 & 94.6 & 90.4 \\
Control & F2b & 98.0 & 91.6 & 89.0 & 91.4 \\
T-I & F2a & 96.4 & 95.8 & 90.8 & 81.6 \\
& F2b & 96.2 & 93.6 & 87.2 & 93.5 \\
T-II & F2a & 97.6 & 98.0 & 96.4 & 73.4 \\
& F2b & 97.0 & 95.7 & 88.0 & 91.6 \\
\hline
\end{tabular}

Live Birth Index $=\frac{\text { Number of Viable Pups Born }}{\text { Total Number of Pups Born }} \times 100$

24-Hour Survival Index $=\frac{\text { Number of Pups Viable at Lactation Day } 1}{\text { Number of Viable Pups Born }} \times 100$

4- Day Survival Index $=\frac{\text { Number of Pups Viable at Lactation Day } 4}{\text { Number of Viable Pups Born }} \times 100$

Lactation Index $=\frac{\text { Number of Pups Viable at Lactation Day } 21}{\text { Number of Pups Retained at Lactation Day } 4} 100$ 
3. Body Weight Data

Body weight data for weanlings are presented in Table $X$. 
TABLE X

TEST MATERIAL: Irradiated Papayas

Three-Generation Repxoduction Study - Albino Rats

Progeny

Wearlung Body Weight Data

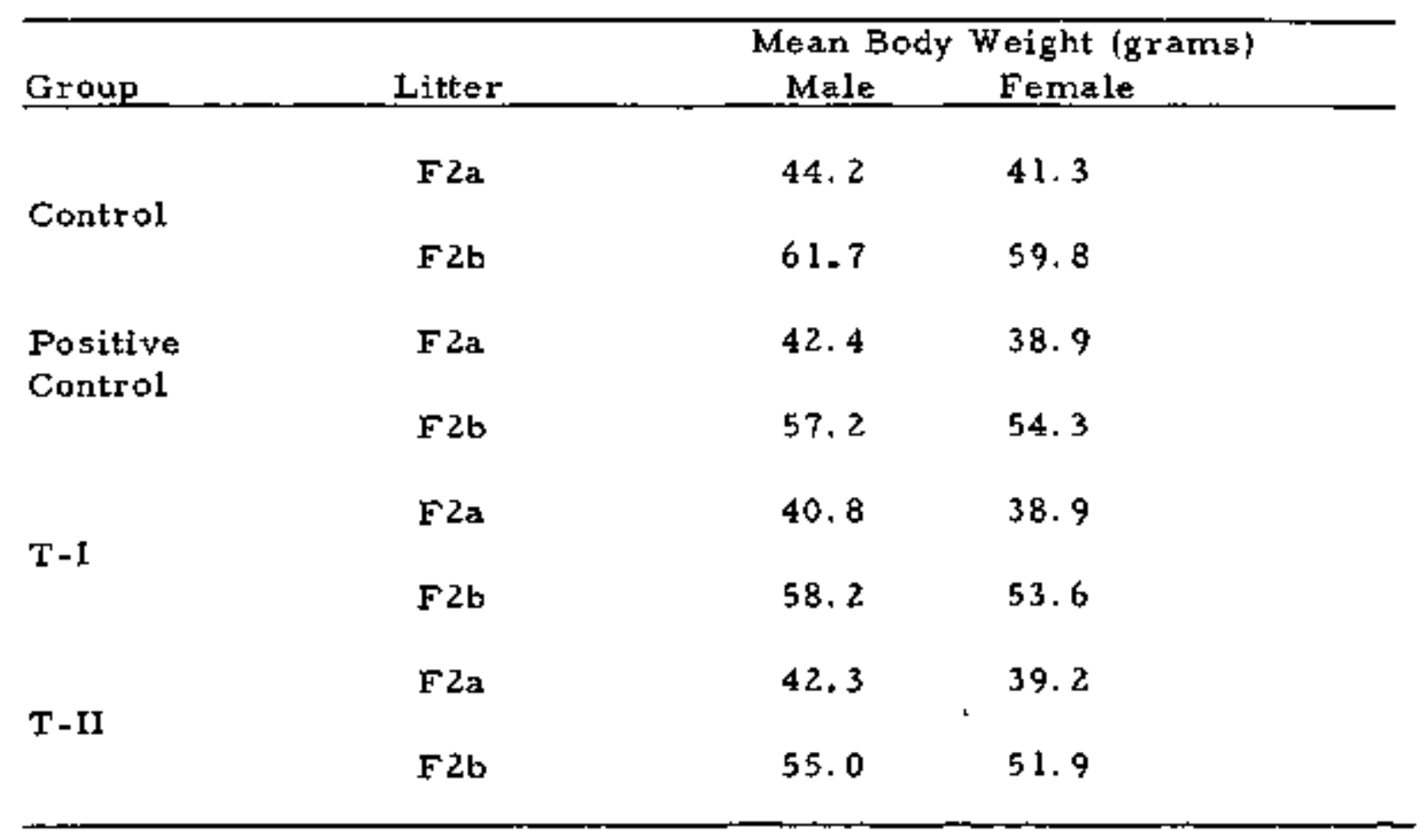


4. Reactions

No untoward reactions were observed among either test or control progeny. 